\title{
Sind die Nistkästen finnisch-ugrisch?
}

Unter Nistkasten verstehen wir hier, wie allgemein in der ethnographischen Literatur, eine für das Eierlegen von bestimmten, in Baumlöchern nistenden Wasservögeln gedachte Nisthöhle, hergestellt aus einem ausgehöhlten oder hohlen Stück Holzstamm, aus Baumrinde oder Brettern, versehen mit Deckel und Boden sowie einer Öffnung im Stammteil; diese Brutkästen werden normalerweise an einem Baum befestigt.

Die Ethnographen haben sich in den skandinavischen Ländern seit Anfang unseres Jahrhunderts, angefangen mit Th. Schvindt $(1905,5)$ mit den Nistkästen beschäftigt, - oft lediglich deskriptiv, doch wurden auch Vermutungen über ihr Alter und ihre Verbreitung angestellt. Als erster hat sich U. T. Sirelius dazu geäussert. In seiner Arbeit "Suomen kansanomaista kulttuuria" (Die volkstümliche Kultur Finnlands) wies er i.J. 1919 darauf hin, dass das Wort uuttu 'Nist-, Brutkasten' auf die fiu. Zeit zurückgeht; er vermutete, weil "ebenfalls die Obugrier wie auch viele andere nördliche Völker solche Nisthöhlen bauen, wird auch das Verfahren selbst auf dieselbe Zeit zurückgehen". (Sirelius 1919, 224-225.) Einige Jahre später wurde diese Auffassung von Sirelius durch E. N. Setälä, vielleicht die bedeutendste Autorität der damaligen nationalen Forschung in Finnland, gewissermassen bestärkt und ' 'kanonisiert", indem er in der Arbeit "Suomen suku" feststellte, dass das Wort uuttu im Finnischen, Lappischen und Ostjakischen begegne und - nach Setäläs Auslegung - ein in einem Baumloch befindliches, teilweise künstliches Wasservogelnest bedeute und dass es sich dabei, "dass die Wasservögel bei 
jedem Volk dieser Sprachen ihre Eier in einem solchen Nest legen, um ein Urerbe handeln könne'. (Setälä 1926, 134.) In dem i.J. 1917 erschienenen, von Setälä verfassten Lexikon-Artikel über die finnisch-ugrischen Völker (Setälä 1917, Spalte 286-294), der Setäläs Text in "Suomen suku" zugrundelag, ist keinerlei Erwähnung über die Nistkästen enthalten, so dass die Ausführungen von Sirelius dazu in seiner "'Suomen kansanomaista kulttuuria" eindeutig den Zusatz veranlasst haben.

Gösta Berg, der i.J. 1933 vor allem die Nistkästen in Skandinavien gründlich untersuchte, sah darin keine finnischugrische Erscheinung, sondern eher ein Beispiel für jene Erscheinungen, die bei der Rekonstruktion der alten gemeinsamen Kulturbasis des nördlichen Europa und Asien als Hilfsmittel herangezogen werden können. (Berg 1933, 118.) Einen sehr vorsichtigen Standpunkt bezog in dieser Hinsicht auch Fr. Leinbock (1934), der Spezialist für die estnischen Nistkästen. Die Dauerhaftigkeit der finnisch-ugrischen Konzeption geht jedoch daraus hervor, dass Toivo Vuorela noch i.J. 1976 poetisch darüber schrieb, wie "schon der Mensch der fernen finnisch-ugrischen Sprachfamilie, von den Gaben der Natur lebend, die von ihren Wanderzügen zurückkehrenden Wasservögel dazu bewegen wollte, in den künstlichen Brutkästen zu nisten, die er in den Bäumen an den Ufern seiner Fischgründe angebracht hatte'. (Vuorela 1976, 74.)

Die Verbreitung der Nistkästen ist nirgends wirklich genauer geklärt worden, vielleicht wegen der Lückenhaftigkeit der Belege. Es liegt jedoch auf der Hand, dass eine Erscheinung wie das Aufstellen solcher Brutkästen an bestimmte naturwissenschaftliche Gegebenheiten gebunden ist, anhand derer zumindest die theoretischen Grenzen im Süden und Norden leicht zu ziehen sind. Im nördlichen Tundragebiet können keine Nistkästen verwendet werden, da dort keine für Nisthöhlen geeignete Bäume wachsen und die betreffenden Vögel nicht soweit nördlich nisten können. Im Süden fällt die Grenze für die Benutzung von Nistkästen entsprechend mit der Verbreitungsgrenze in Baumhöhlen nistender Wasservögel zusammen. Am südlichsten nistet die Schellente (Bucephala clangula), deren Nistgebietgrenze ungefähr in der Höhe der Südküste der Ostsee 
verläuft: das Nistgebiet von Zwergsäger (Mergus albellus) und Gänsesäger (Mergus merganser) liegt noch nördlicher. (Heinzel u.a. 1974, 64-66.) Andere als diese drei Gattungen, die reichlich Eier legen und die Anzahl ihrer Eier leicht vermehren, kommen praktisch für die Nistkästen gar nicht in Frage.

Innerhalb dieser naturwissenschaftlichen Grenzen liegen auch die faktischen Angaben über die Verwendung von Nistkästen, die allerdings, was besonders die nördlichen Bereiche von Russland angeht, sichtlich lückenhaft sind. Ohne hier die Nachrichten und Quellen über die Nistkästen genauer zu spezialisieren, kann doch allgemein festgestellt werden, dass von Westen an genannt - wenigstens die Norweger, Schweden, Lappen, Finnen, Esten, Karelier und Syrjänen solche Brutkästen benutzt haben. Damit scheinen aber auch die Angaben im Osten aufzuhören: vom Ural und aus den Gebieten jenseits dieses Gebirges liegen keine Belege dafür vor. Allerdings haben z.B. die Obugrier die Eier von Wasservögeln in deren eigenen Nestern gesammelt (Prokof'yeva u.a. 1963, 523), und z.B. von den Wogulen an der Lozva wird berichtet, dass sie ausser Hühnereiern auch Birkhuhneier verzehrten (Gluškov 1900, 41), was natürlich eine entsprechende Sammeltätigkeit voraussetzt. Nistkästen werden jedoch in keinem Zusammenhang erwähnt, obwohl das doch angenommen werden könnte, falls sie bekannt gewesen wären.

Am überraschendsten ist vielleicht, dass Sirelius, der als erster die Hypothese vom finnisch-ugrischen Nistkasten aufstellte, in seinen umfangreichen obugrischen Aufzeichnungen, die er in den Jahren 1898-1900 machte, keine einzige Angabe über das Nisten in Brutkästen bringt. Die einzige Angabe über Eier berichtet über das Sammeln von Enteneiern in den Jurten Tsingalinskija am Irtysch. (Sirelius III, 887.)

Dieser Widerspruch zwischen den Aufzeichnungen von Sirelius und seinen späteren Vermutungen über die Nistkästen kann darauf zurückgehen, dass der Sprachforscher Heikki Paasonen - ein guter Freund von Sirelius - i.J. 1906 nachwies, dass das Wort uuttu auf die fiu. Zeit zurückgeht und wenigstens im Ostjakischen eine Entsprechung hat. (Paasonen 1906, 241.) Das Wort bedeutete allerdings in keinem der von Paasonen ange- 
führten ostjakischen Fälle einen künstlich hergestellten Nistkasten, sondern es bezeichnete nur eine Vertiefung oder Höhle in einem Baum und in einem Fall - im Dialekt vom Kazym das Nest eines Wasservogels im Baum. Diese lexikalischen Verbindungen waren jedoch für Sirelius, dem an einer Rekonstruktion einer finnisch-ugrischen Urkultur gelegen war, so verlockend, dass er sich erinnern wollte, Nistkästen im obugrischen Gebiet gesehen zu haben oder wenigstens seine Aufzeichnungen über das Sammeln von Eiern ausdrücklich mit diesen aufgestellten Nistkästen in Verbindung bringen wollte. Schliesslich wirkte die Erscheinung "primitiv" genug, um Jahrtausende alt zu sein.

Dass eine Kulturerscheinung wie die Benutzung von Nistkästen nicht jenseits des Urals bekannt ist, wirkt insofern nicht überraschend, als schon etwas weiter östlich das Sammeln von Eiern aus den von den Vögeln selbst in der Natur gebauten Nestern aufhört. Die Samojeden haben zwar sowohl in Europa als auch in Sibirien Eier gesammelt (u.a. Homič 1966, 135; Žitkov 1913, 210), und auch von den Nganasanen (Popov 1966, 113) und Dolganen (Popov 1937, 179) liegen Angaben über das Sammeln von Eiern vor; weiter östlich fehlt jegliche Nachricht darüber. Man könnte natürlich meinen, dass es sich lediglich um eine Lückenhaftigkeit des Materials handle, doch angesichts jener Genauigkeit, mit der besonders Kerstin Eidlitz (1969) die Schilderungen über Sibirien für ihre volkskundliche Untersuchung der Nahrungswirtschaft durchgesehen und keine Erwähnung über das Sammeln und die Verwendung von Eiern in den mittleren und östlichen Teilen Sibiriens gefunden hat, wirkt das nicht wahrscheinlich. Erst unmittelbar in der Nähe des Stillen Ozeans sind wieder Eier gesammelt worden, und von hier aus reicht dann auch das Verwendungsgebiet der Eier zusammenhängend bis nach Nordamerika und zu den Eskimos. (Eidlitz 1969, 39-40.)

Überall wo Nistkästen in Gebrauch sind, ist auch "normal" gesammelt worden, d.h. man hat die eigenen Nester der Vögel ausgenommen, welches Vorgehen auch verbreitet südlich des Beleggebietes der Nistkästen bekannt war sowie auch im nördlichen Tundragebiet. Die Verwendung von Nistkästen ist 
denn auch eine Art spezialisiertes Sammelverfahren, das sich innerhalb des Gebietes abhebt, in dem Eier gesammelt werden: entsprechende spezialisierte, aus dem Sammeln hervorgegangene Methoden gibt es noch andere, wie z.B. die Verwendung eines Stöckchens, das zwecks Erhöhung der Eierzahl der Vögel ins Nest getan wird. (S. Lehtonen 1970.) In diesem nordeuropäischen Gebiet ist die Verwendung von Brutkästen eindeutig entstanden. Es erscheint dagegen nicht möglich, das Phänomen speziell mit den finnisch-ugrischen Völkern zu verbinden und es auf die finnisch-ugrische Zeit zu datieren.

Im Lichte der sprachwissenschaftlichen Beweise ist allerdings klar, dass das Nisten in Brutkästen bereits in urfinnischer Zeit bekannt war. Die zur Sippe koskelo 'Gänsesäger' gehörenden Vogelnamen in den ostseefinnischen Sprachen gehen nach R. E. Nirvi (1941, 338) nämlich darauf zurück, dass man für diese Wasservögel Nester aus Baumrinde (fi. koskut) herstellte. Berücksichtigt man die Allgemeinheit der Nistkästen speziell in Skandinavien (Berg 1933) und in den schwedischen Gegenden von Finnland (Andersson 1942) und Estland (Leinbock 1934), kann die Möglichkeit nicht ausgeschlossen werden, dass diese Überlieferung wenigstens in Finnland und Estland östliche und westliche Schichten verschiedenen Datums aufweist. (S. auch Leinbock 1934, 180.)

Es ist schwer zu sagen, in welcher Beziehung die NistkastenTradition der Syrjänen zu jener im Ostseeraum steht: möglich ist jedoch, dass hier eine alte Kulturerscheinung vorliegt, deren Gebiet einheitlich bis nach Ostrussland reichte, und die durch den Einfluss der russischen Bevölkerung, die aus dem Gebiet südlich der Nistkastenzone eintraf, verdrängt wurde. Aufschlussreich ist jedenfalls die Feststellung, dass Nistkästen in den syrjänischen Gebieten sowohl an der Sysola (Mihajlov 1851,98 ) als auch am Oberlauf der Petschora (Hofmann 1856, 6) angetroffen worden sind, was bedeuten könnte, dass der Nistkasten mit den Siedlungsbewegungen des 18. Jh. (s. Lašuk 1958, 112-6) in das letztgenannte Gebiet gelangte.

Die Kulturgrenze, die die sibirischen Kulturen von der europäischen trennte (s. Bacon 1946; Istoriko-ètnografičeskij atlas Sibiri), haben die Nistkästen sichtlich niemals überschrei- 
ten können. Die Kulturgrenze, die in der Hauptsache den Ural entlang verläuft, im Norden im Gefolge der samojedischen Kulturen weiter nach Westen ausholt und im Süden des Urals in Richtung Osten eine breitere Übergangszone ergibt, war gerade in jenen Breitengraden besonders scharf, wo auf europäischer Seite Nistkästen haben auftreten können.

Man hat das Sammeln von Eiern und die Benutzung von Nistkästen auch mit primitiven und direkt aus früheren Kulturperioden stammenden reliktartigen Erscheinungen in Verbindung gebracht: "surviving primitive gathering". (Z.B. Erixon 1951, 95.) In der ersten Hälfte unseres Jahrhunderts herrschte denn auch im Bereich der Ethnologie die Auffassung, dass gerade derartige Wirtschaftsformen auf alte Kulturepochen zurückgingen. Nach der evolutionistischen Denkweise gab es Survivals, die sich als seltene Beweisstücke aus der Vergangenheit bewahrt hatten, während sich die Kultur in ihren anderen Erscheinungsformen weiterentwickelte.

Auch die Verwendung von Nistkästen ist, daran besteht kein Zweifel, eine alte Relikterscheinung. (S. Storå 1966, 195.) Doch das bedeutet nicht, dass sie - ebensowenig wie auch die anderen Formen des Sammelns - ein Relikt der eigentlichen Sammelwirtschaft wäre. In jenen relativ nördlichen Gebieten, wo die Nistkästen überhaupt Verwendung finden konnten, hat eine reine Sammelwirtschaft nie die vorherrschende Wirtschaftsform oder Grundlage des Unterhalts bilden können. Natürlich hat man neben der Jagd oder der Landwirtschaft immer auch gesammelt (s. Gjessing 1955, 58-60), doch stets nur zur Ergänzung des Nahrungshaushalts anderen Typs (s. Grøn 1942, 57); dass das Sammeln im Grunde auch eine ganz junge Erscheinung sein kann, geht z.B. daraus hervor, dass sich das Pilzesammeln erst seit dem ausgehenden 19. Jh. in Finnland durchsetzte. (Talve 1973, 81-5.) Das Sammeln überhaupt war also eine vom übrigen Nahrungshaushalt losgelöste Art der Nahrungsbeschaffung, und auch die Eier, die man aus den Nistkästen holte, waren nur eine besondere Delikatesse im Frühjahr und im Vorsommer, und zwar in gleicher Weise für den Bauern wie für den Fischer und den Rentierzüchter. 